\title{
NUMERICAL SOLUTION OF BLOOD DYNAMICS IN ATHEROSCLEROTIC CAROTIDS
}

\author{
CHRISTIAN VERGARA (*) \\ Nota presentata dal m.e. Carlo Domenico Pagani \\ (Adunanza del 23 giugno 2016)
}

SunTO. - In questo lavoro verrà discussa la modellazione matematico-numerica della fluido-dinamica del sangue in carotidi aterosclerotiche. Verranno richiamati i concetti di base per la ricostruzione delle geometrie computazionali, la modellazione matematica del problema di interazione-fluido struttura e una possibile strategia per la sua risoluzione numerica. Verranno inoltre presentati alcuni risultati numerici ottenuti per un caso sia prima che dopo la rimozione chirurgica della placca.

$* * *$

ABSTRACT. - In this work we present the mathematical and numerical modeling of blood fluid-dynamics in atherosclerotic carotids. We review how to obtain the computational domain, the mathematical formulation of the fluid-structure interaction problem, and a possible strategy for its numerical discretization. We also report some numerical results for a case both before and after the surgical removal of the plaque.

\section{INTRODUCTION}

Atherosclerosis is the leading cause of death in Western countries and consists in the formation of atherosclerotic plaques in arteries of medium or large size, with possible obstruction of the vessel and formation of blood clots [18]. Under normal conditions, this process is a protective response of the endothelium and muscle cells of the artery to stress, and consists in the formation of fibrous lesions. When this

(") LABS - Dipartimento di Chimica, Materiali e Ingegneria Chimica "Giulio Natta” - Politecnico di Milano, Italia. E-mail: christian.vergara@polimi.it 
response becomes excessive as a result of prolonged and high fluid viscous stresses, a matrix of connective tissue grows up and lipids begin to accumulate. This accumulation can lead to the formation of a plaque, that obstructs the vessel (stenosis), reducing its lumen, and could degenerate leading to a complete obstruction of the vessel and to a stroke.

The plaque may be calcified, fibrous or lipid, depending on its composition, and mostly develops at the bifurcation sites, such as the carotid one. The carotids are two arteries located at the level of the neck and are crucial to maintain the human life, since they supply the blood to the brain. In the case of a lipid plaque in the carotids, the thin fibrous cap may crumble inducing embolization of fragments into the brain tissue.

The mechanisms of formation and progression of the atherosclerotic plaque are related to several factors, such as vessel morphology and vascular cell biology, interacting with each other. In particular, the blood fluid-dynamics seems to play a crucial role in the plaque development. Indeed, it has been extensively shown in the last thirty years that when vascular endothelial cells are subjected to stress, they change their polygonal shape and become elongated in the direction of the blood flow. A number of molecular and biological changes are mediated by shear stress exposure of endothelial cells, including changes in gene expression, production of vaso-active compound, adhesion molecules and changes in cell cytoskeleton. As a result of this biological response to flow, the intact endothelium is able to initiate a cascade of events leading to the thickening of the arterial wall and to plaque formation in regions of low and oscillating wall shear stress (WSS), whereas areas of vessel wall characterized by uniformly directed and laminar WSS are spared from development of vascular disease [11]. All these phenomena, induced by the blood fluid-dynamics, are highly correlated to the formation and progression of the plaque, and should be considered in the study of these clinical conditions, not only in general terms but at patient-specific level.

The mathematical and numerical modeling is nowadays a valuable tool for the study and the understanding of many physical phenomena. In particular, the use of numerical approximations of the equations describing these phenomena is a synergistic instrument (in some cases even alternative) to experimental studies. There are many areas in which the numerical modeling significantly supports the understanding of physical phenomena and the industrial design, such as aerodynamics, shipbuilding, geo-science, structural mechanics, electronics 
and, most recently, the biomedical environment. In the context of patient-specific numerical simulations for the study of the cardiovascular system, the research has been active for at least twenty years, see, e.g., [15]. In addition, the opportunities given by modern computers and high performance computing allow to achieve meaningful patientspecific results in a reasonable time. This has been also possible thanks to the great advances in recent years of the techniques to obtain biomedical data and imaging and the related techniques to reconstruct geometries and process data, which are a necessary starting point in view of the numerical studies.

In this work, we present the numerical solution of blood flow in carotids. To this aim we consider the fluid-structure interaction problem due to the compliant nature of the carotid vessel wall and its numerical discretization. In particular, we address the case of stenotic carotids, so that we need to introduce a suitable model for the plaque. We present some numerical results obtained in realistic geometries and with patient-specific boundary conditions, both before and after the plaque removal.

\section{GenERATION OF THE COMPUTATIONAL DOMAINS}

The equations that describe blood flow in a compliant vessel (see Cap. 3 for their formalization) should be numerically solved in a realistic computational domain. This is mandatory to obtain significant results from a clinical perspective. To do this, the following steps should be performed: i) reconstruction of the lumen geometry, ii) deflation of the domain, iii) generation of the vessel wall domain, and iv) generation of the computational meshes.

Reconstruction of the lumen geometry. The reconstruction of the lumen geometry provides a first guess of the domain where the fluid problem will be solved. To do this, a standard procedure is based on the segmentation of medical images based on Magnetic Resonance Imaging (MRI) or Computed Tomography (CT) technology. Since these images are often affected by noise, an imaging enhancement is usually performed at the beginning of the procedure $[19,16,6]$. Then, image segmentation starts. This consists in the construction of the shape of the vascular district, by detecting those points which (presumably) belong to the boundary of the vessel lumen (i.e. to the interface with the vessel 
wall). Typically, a thresholding technique is used, where a threshold $k$ is selcted to identify the points whose intensity in grey-scale is larger than $k$. This is motivated by the assumption that the vessel lumen is characterized by intensity values larger than the background [1]. More sophisticated class of segmentation methods are front propagation methods, where the propagation of a suitable wavefront is tracked, based on noticing that the speed of the wave is small in regions where the image intensity changes rapidly, so that the wavefront is supposed to slow down when approaching the boundary [22]. See [17] for a review of other methods. In Fig. 1, an example of final reconstruction of an ascending aorta is provided.

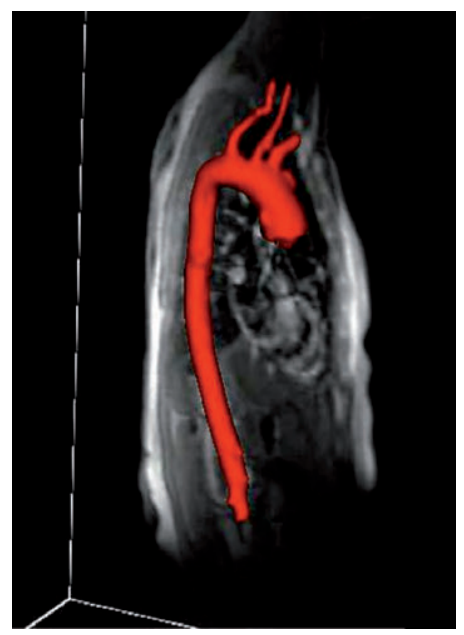

Fig. 1 - Example of segmentation of the lumen boundary of an ascending aorta. Courtesy of E. Faggiano.

In this work, we consider MRI images of the carotid of a patient who underwent to carotid endarterectomy (TEA) (i.e. the surgical plaque removal) both before (i.e. in the stenotic case) and after (i.e. without plaque) TEA. These data have been provided by the Ospedale Maggiore Policlinico di Milano. The segmentation of such geometries has been performed with the open source software VMTK (www.vmtk.org).

Deflation of the computational domain. In this work, we consider a non-linear elastic material (see Cap. 3), so that we could not ignore the non-null diastolic blood pressure which characterizes a radiological image. This means that the geometry we have reconstructed, corre- 
sponds to an internal pressure of about $70 \mathrm{mmHg}$. To account for this, here we use the strategy introduced in $[12,3]$. In particular, the idea is to recover the zero-stress geometry (that is the one would have without the blood inside the lumen) by suitably deflating the diastolic one. This is obtained by introducing an inverse problem that has been solved by means of fixed point iterations (see [12] for more details). In Fig. 2 the deflation of a carotid domain is shown.
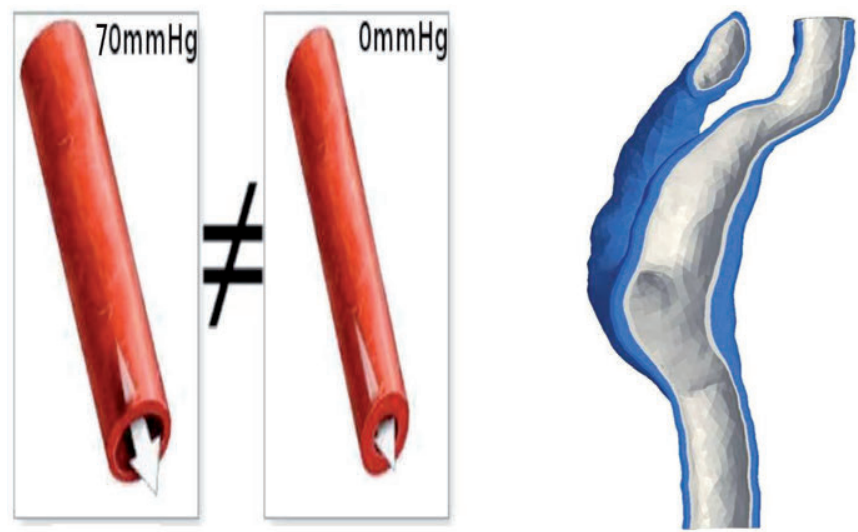

Fig. 2 - Deflation of a carotid.

In blue the original segmentation, in grey the geometry after the deflation.

Generation of the structure domain. The vessel wall is not detectable from standard radiological images. Instead, the lumen where blood flows and the plaque could be identified. Once the lumen geometry has been reconstructed (see previous point), the region occupied by the plaque is reconstructed as well (see Fig. 3, left). Thus, the vessel wall domain was obtained by extrusion, from the surface delimiting the lumen/plaque reconstruction, with a total wall thickness equal to $20 \%$ of the local vessel radius, intended as the sum of the lumen and plaque transversal distance from the center [4], see Fig. 3, left.

Generation of the computational meshes. Once the three geometries characterizing the different part of a carotid (the lumen where blood flow, the plaque, and the vessel wall) have been reconstructed, the internal volume of these regions were discretized using tetrahedral elements.

In Fig. 3, middle and right, we report the computational meshes of the lumen vessel after deflation considered in this work, both before and after TEA. 

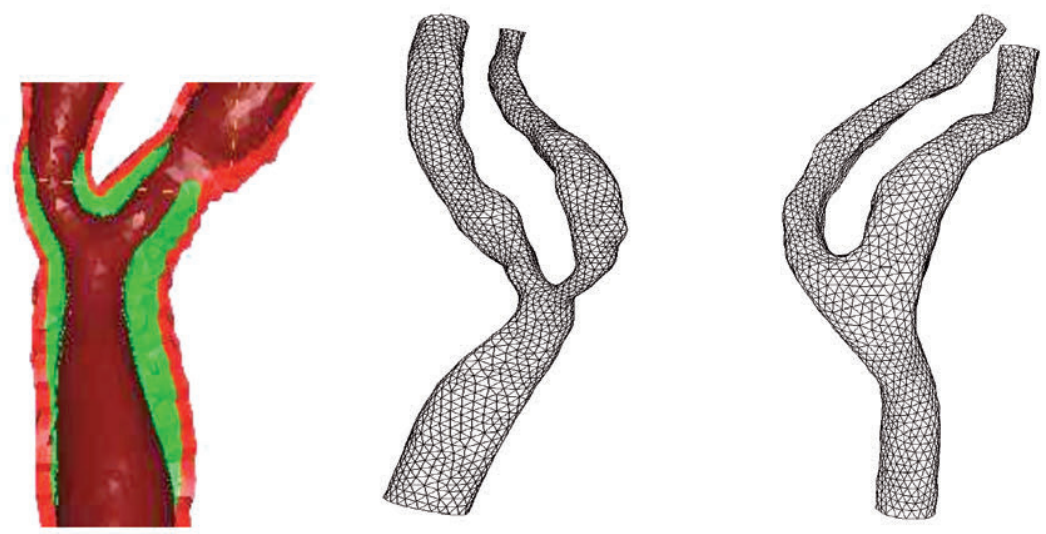

Fig. 3 - Left. Reconstruction of the lumen/plaque/vessel wall geometry.

In green, the plaque; in red, the vessel wall obtained by extrusion.

Middle and right. Computational meshes for the fluid problem before the plaque removal (stenotic case) and after the plaque removal, respectively.

\section{MATHEMATICAL AND NUMERICAL MODELING}

\subsection{Mathematical modeling}

As highlighted in the Introduction, blood flows in compliant vessels. From a mathematical point of view, this can be described by a fluid-structure interaction (FSI) problem.

Well accepted hypotheses for blood are incompressibility and homogeneity. Moreover, its rheology could be considered Newtonian for medium and large vessels such as carotids [15]. The deformation of the vessel wall is mathematically described by the elastodynamics equation. It is often assumed a non-linear finite elastic law which relates the stress tensor to the strain, and a nearly incompressible behavior [10].

The fluid problem is usually written in an Eulerian framework, whereas the vessel wall problem in a Lagrangian configuration. Given a function $g$ defined in the current configuration $\Omega^{t}$, we denote by $\hat{g}=g \circ \mathcal{L}$ the function in the reference configuration $\Omega, \mathcal{L}$ being the Lagrangian map. Moreover, $F=\nabla x$ is the deformation tensor and $J=\operatorname{det}(\boldsymbol{F})$ represents the change of volume between the reference and the current configuration. The stress tensor in the reference configuration (First Piola-Kirchhoff stress tensor) is mapped into the current con- 
figuration (Cauchy stress tensor), as follows: $\hat{T_{s}}=J T_{s} \boldsymbol{F}^{-T}$. The common boundary between the two regions is the fluid-solid (FS) interface $\Sigma^{t}$, which coincides with the physical lumen boundary and the internal vessel wall/plaque boundary, see Fig. 4, left.

Thus, the FSI problem at each time reads as follows:

$$
\begin{array}{ll}
\rho_{f}\left(\frac{\partial \boldsymbol{v}}{\partial t}+(\boldsymbol{v} \cdot \nabla) \boldsymbol{v}\right)-\nabla \cdot \boldsymbol{T}_{f}(\boldsymbol{v}, p)=\mathbf{0} & \text { in } \Omega_{f}^{t}\left(\boldsymbol{d}_{f}\right), \\
\nabla \cdot \boldsymbol{v}=0 & \text { in } \Omega_{f}^{t}\left(\boldsymbol{d}_{f}\right), \\
\boldsymbol{v}=\frac{\partial \boldsymbol{d}}{\partial t} & \text { at } \Sigma^{t}\left(\boldsymbol{d}_{f}\right), \\
\boldsymbol{T}_{s}(\boldsymbol{d}) \boldsymbol{n}=\boldsymbol{T}_{f}(\boldsymbol{v}, p) n & \text { at } \Sigma^{t}\left(\boldsymbol{d}_{f}\right), \\
\rho_{s} \frac{\partial^{2} \widehat{\boldsymbol{d}}}{\partial t^{2}}-\nabla \cdot \widehat{\boldsymbol{T}}_{s}(\widehat{\boldsymbol{d}})=\mathbf{0} & \text { in } \Omega_{s}, \\
\boldsymbol{d}_{f}=\boldsymbol{d} & \text { at } \Sigma^{t},
\end{array}
$$

where $v$ is the blood velocity, $p$ the blood pressure, $T_{f}=1 / 2 \mu\left(\nabla \boldsymbol{u}+\nabla \boldsymbol{u}^{T}\right)$ $-p I$ the Cauchy fluid stress tensor, $\mu$ the blood viscosity, $d$ the vessel wall displacement, and $\boldsymbol{d}_{f}$ the fluid domain displacement; $\rho_{f}$ and $\rho_{s}$ denote the fluid and vessel wall densities. $\hat{T}_{s}\left(\hat{\eta_{s}}\right)$ is the first Piola-Kirchhoff tensor for a nearly incompressible exponential material, that is

$$
\widehat{\boldsymbol{T}}_{s}(\boldsymbol{F})=G J^{-2 / 3}\left(\boldsymbol{F}-\frac{1}{3} \operatorname{tr}\left(\boldsymbol{F}^{T} \boldsymbol{F}\right) \boldsymbol{F}^{-\mathrm{T}}\right) e^{\gamma\left(J^{-\frac{2}{3}} \operatorname{tr}\left(\boldsymbol{F}^{T} \boldsymbol{F}\right)-3\right)}+\frac{\kappa}{2}\left(J-1+\frac{1}{J} \ln (J)\right) J \boldsymbol{F}^{-\mathrm{T}},
$$

where $\kappa$ is the bulk modulus, $G$ the shear modulus, and $\gamma$ regulates the exponential behaviour. For small deformations this material behaves as a linear structure. In order to accout for the plaque, we will consider different values of $\kappa$ and $G$ in the region of the vessel wall and in that of the plaque, accounting for the increased stiffness in the latter, see Fig. 4, right.
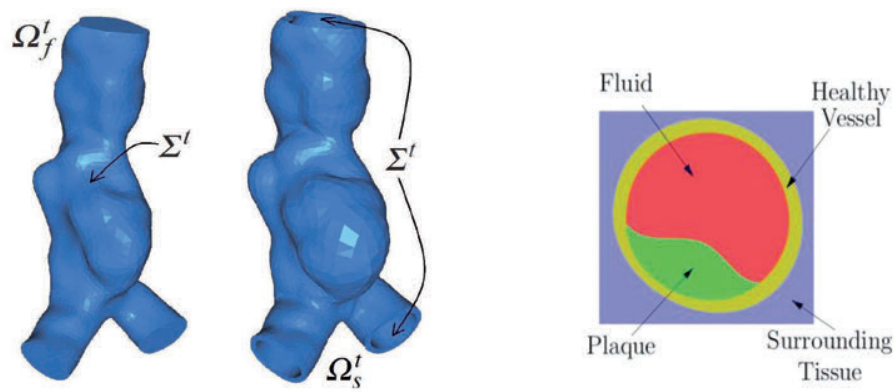

Fig. 4 - Left. Fluid and structure domains.

Right. schematic representation of the different computational domains depicted through a section. 
In the coupled problem above, together with the Navier-Stokes equations (1a)-(1b) and the elastodynamics equation (1e), three interface coupling conditions are identified: the kinematic condition (1c) stating a no-slip assumption between fluid and structure particles; the dynamic condition (1d) expressing the action-reaction principle (III Newton law); and the geometric condition (1f) enforcing the perfect adherence between fluid and structure domains.

Problem (1) should be completed with suitable initial condition for $v, d$ and $\dot{d}$, and boundary conditions. For the structure, here we consider homogeneous Dirichlet and Neumann conditions at the artificial sections in the normal and tangential directions, respectively, and a Robin condition with coefficient $\alpha_{e}$ to account for the presence of the surrounding tissue at the external surface [17]. For the fluid problem, we prescribe a flow rate at the inlet and an absorbing resistance condition at the outlet [14].

\subsection{Numerical approximation}

For the numerical solution of the FSI problem (1), segregated algorithms are here considered, where the solution of the fluid and structure problems are solved separately in an iterative way.

After time discretization (BDF1 for the fluid and mid-point scheme for the structure), the fluid problem is linearized by means of a semi-implicit approach, where the convective term and the fluid domain are extrapolated from previous time steps. This choice introduces a (mild) CFL-like restriction on the time step $\Delta t$ to preserve absolute stability. We indicate with * extrapolated quantities. Given $\sigma_{f} \neq \sigma_{s}$, a general segregated algorithm reads as follows: $k=k_{\max }$,

For $n \geq 1, k \geq 1$, at time step $t^{n}$ /iteration $k$, until convergence or

1. solve the Oseen problem with a Robin condition at the FS interface:

$$
\begin{array}{ll}
\frac{\rho_{f}}{\Delta t} \boldsymbol{v}_{(k)}^{n}+\rho_{f}\left(\left(\boldsymbol{v}^{*}-\boldsymbol{v}_{f}^{*}\right) \cdot \nabla\right) \boldsymbol{v}_{(k)}^{n}-\nabla \cdot \boldsymbol{T}_{f}\left(\boldsymbol{v}_{(k)}^{n}, p_{(k)}^{n}\right)=\boldsymbol{g}_{f}^{n} & \text { in } \Omega_{f}^{*}, \\
\nabla \cdot \boldsymbol{v}_{(k)}^{n}=0 & \text { in } \Omega_{f}^{*}, \\
\sigma_{f} \boldsymbol{v}_{(k)}^{n}+\boldsymbol{T}_{f}\left(\boldsymbol{v}_{(k)}^{n}, p_{(k)}^{n}\right) \boldsymbol{n}^{*}=\sigma_{f}\left(\frac{1}{\Delta t} d_{(k-1)}^{n}+\boldsymbol{g}_{f s}^{n}\right)+\boldsymbol{T}_{s}\left(\boldsymbol{d}_{(k-1)}^{n}\right) \boldsymbol{n}^{*} & \text { on } \Sigma^{*} ;
\end{array}
$$

2. then, solve the non-linear vessel wall problem with another Robin condition at the FS interface: 


$$
\begin{array}{ll}
\frac{\rho_{s}}{\Delta t^{2}} \widehat{\boldsymbol{d}}_{(k)}^{n}-\nabla \cdot \widehat{\boldsymbol{T}}_{s}\left(\widehat{\boldsymbol{d}}_{(k)}^{n}\right)=\widehat{\boldsymbol{g}}_{s}^{n} & \text { in } \Omega_{s}, \\
\frac{\sigma_{s}}{\Delta t} \widehat{\boldsymbol{d}}_{(k)}^{n}+\widehat{\boldsymbol{T}}_{s}\left(\hat{\boldsymbol{d}}_{(k)}^{n}\right) \widehat{\boldsymbol{n}}=\sigma_{s} \widehat{\boldsymbol{v}}_{(k)}^{n}+\widehat{\boldsymbol{T}}_{f}\left(\widehat{\boldsymbol{v}}_{(k)}^{n}, \hat{p}_{(k)}^{n}\right) \widehat{\boldsymbol{n}}-\sigma_{s} \widehat{\boldsymbol{g}}_{f s}^{n} & \text { on } \Sigma,
\end{array}
$$

where $v_{f}=d_{f}$ and the terms $g$ account for the quantities at previous time steps. For the choice of the interface parameters $\sigma_{f}$ and $\sigma_{s}$, several choices have been proposed to obtain fast convergence, see e.g. [2, 7, 8, 21].

At each iteration the fluid problem is solved in a (known) moving domain $\Omega^{*}$. This corresponds to an explicit treatment of the geometric interface condition (1f). This choice produces stable results in hemodynamics, due to the restrained wall displacements [5]. For its solution, we consider the Arbitrary Lagrangian-Eulerian configuration, i.e. we write the fluid equations in a framework moving with the fluid domain [9]. For the reconstruction of the latter, an harmonic extension of the geometric interface condition is adopted. On the other side, the vessel wall problem is linearized by means of the Newton method.

\section{NUMERICAL RESULTS}

We present here some numerical results obtained in the two geometries described above, namely the carotid of a patient before and after the removal of the atherosclerotic plaque. These numerical results were obtained using the Finite Elements library LifeV (www.lifev.org), $P 1$ - Bubble/P1 Finite Elements for the fluid problem and P1 Finite Elements for the vessel wall/plaque problem; the backward Euler scheme and the midpoint method have been used for the time discretization of the fluid and vessel wall/plaque problems, respectively. The implicit Robin-Robin partitioned scheme has been used for the solution of the FSI problem. For the choice of the interface parameters $\sigma_{f}$ and $\sigma_{s}$, we used the values proposed in $[2,13]$, which guarantee excellent convergence properties.

We also set $\rho_{f}=1.0, \mathrm{~g} / \mathrm{cm}^{3}, \rho_{\mathrm{s}}=1.1 \mathrm{~g} / \mathrm{cm}^{3}, \mu=0.035 \mathrm{~cm}^{2} / \mathrm{s}$ For the healthy vessel wall, we set $\kappa=10^{7}$ dyne/ $\mathrm{cm}^{2}, G=1.034 \cdot 10^{6} \mathrm{dyne} / \mathrm{cm}^{2}$ (corresponding for small displacements to $E=3 \cdot 10^{6} \mathrm{dyne} / \mathrm{cm}^{2}$ and $v=$ $0.45), \gamma=1$, whereas for the plaque $\kappa=6.67 \cdot 10^{7}$ dyne/ $\mathrm{cm}^{2}, G=6.897$ $\cdot 10^{6}$ dyne $/ \mathrm{cm}^{2}$ (corresponding for small displacements to $E=2 \cdot 10^{7}$ dyne $/ \mathrm{cm}^{2}$ and $\left.v=0.45\right), \gamma=1$. The time discretization parameter was $\Delta t$ $=0.002 \mathrm{~s}$. 
In Fig. 5, left, we show the flow rate prescribed at the inlet and obtained by means of Echo-Doppler measurements at Ospedale Maggiore Policlinico di Milano. This datum has been prescribed by means of a Dirichlet condition obtained by selecting a parabolic profile for the velocity. At the outlet, a resistance condition to avoid spurious reflections has been prescribed, see [14]. In Fig. 5, middle, the computed pressures at the level of the bifurcation has been reported both before and after the plaque removal. From this comparison, we observe the reduced pressure obtained after the removal of the plaque, in accordance with the clinical evidences. In Fig. 5, right, the computed blood velocity streamlines together with the vessel wall/plaque displacements (in arrows) at the systolic peak have been reported.
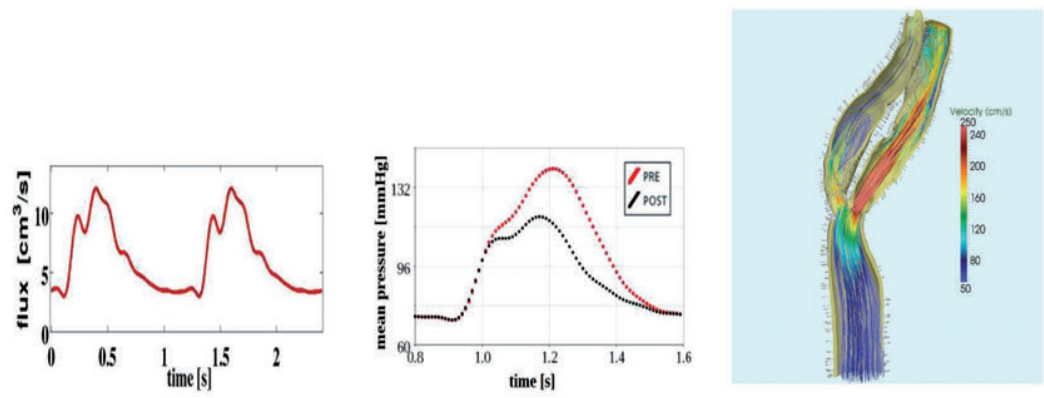

Fig. 5 - Left. flow rate prescribed at the inlet obtained by means of Echo-Doppler measurements; Middle. Computed pressure at the level of the bifurcation;

Right. Computed blood velocity stream-lines and vessel wall/plaque displacements at the systole.
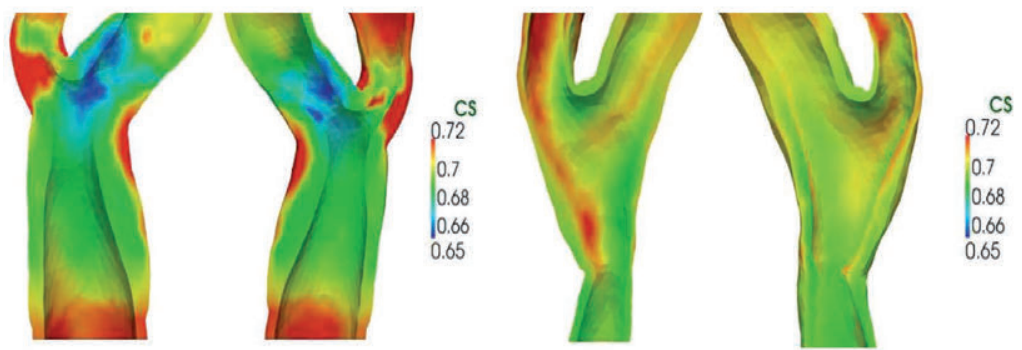

Fig. 6-Cyclic strain before (left) and after (right) the plaque removal.

In Fig. 6, we report the cyclic strain in the vessel wall/plaque domain both before and after the plaque removal. This quantity is 
defined for each point as $\left(V_{\text {syst }}-V_{\text {diast }}\right) / V_{\text {syst }}$, where $V_{\text {syst }}$ and $V_{\text {diast }}$ are the systolic and diastolic Von Mises stress [20]. This quantity seems to be correlated with high values of the permeability of the wall which should favour the plaque development. We observe the decrease of the cyclic strain after the plaque removal.

\section{ACKNOWLEDGEMENTS}

The author would like to thank M. Domanin, E. Faggiano, R.M. Lancellotti, and M. Pozzoli.

\section{REFERENCES}

[1] L. Antiga, J. Peiro, and D.A. Steinman. From image data to computational domains. In Cardiovascular mathematics, edited by L. Formaggia, A. Quarteroni, A. Veneziani, Chapter 4, pages 123-175. Springer, 2009.

[2] S. Badia, F. Nobile, and C. Vergara. Fluid-structure partitioned procedures based on Robin transmission conditions. Journal of Computational Physics, 227:7027-7051, 2008.

[3] J. Bols, J. Degroote, B. Trachet, B. Verhegghe, P. Segers, and J. Vierendeels. A computational method to assess the in vivo stresses and unloaded configuration of patient-specific blood vessels. Journal of Computational and Applied Mathematics, 246:10-17, 2013.

[4] E. Faggiano, L. Formaggia, and L. Antiga. An open-source tool for patient-specific fluid-structure vessel mesh generation. In $V$ International Symposium on Modelling of Physiological Flows (MPF), pages 25-26, 2013.

[5] M.A. Fernandez, J.F. Gerbeau, and C. Grandmont. A projection semi-implicit scheme for the coupling of an elastic structure with an incompressible fluid. International Journal for Numerical Methods in Engineering, 69(4):794-821, 2007.

[6] A.F. Frangi, W.J. Niessen, R.M. Hoogeveen, T. Van Walsum, and M.A. Viergever. Model-based quantitation of $3-\mathrm{d}$ magnetic resonance angiographic images. IEEE Transactions on Medical Imaging, 18(10):946-956, 1999.

[7] L. Gerardo Giorda, F. Nobile, and C. Vergara. Analysis and optimization of robin-robin partitioned procedures in fluid-structure interaction problems. SIAM Journal on Numerical Analysis, 48(6):2091-2116, 2010.

[8] G. Gigante and C. Vergara. Analysis and optimization of the generalized schwarz method for elliptic problems with application to fluid-structure interaction. Numer. Math., 131(2):369-404, 2015.

[9] C.W. Hirt, A.A. Amsden, and J.L. Cook. An arbitrary lagrangian eulerian computing method for all flow speeds. Journal of Computational Physics, 69:277-324, 1974. 
[10] G.A. Holzapfel and R.W. Ogden. Constitutive modelling of arteries. Proc. R. Soc. Lond. Ser. A Math. Phys. Eng. Sci., 466(2118):1551-1596, 2010.

[11] D.N. Ku, D.P. Giddens, C.K. Zarins, and S. Glagov. Pulsatile flow and atherosclerosis in the human carotid bifurcation. positive correlation between plaque location and low oscillating shear stress. Arteriosclerosis, 5(3):293-302, 1985.

[12] R.M. Lancellotti. Numerical Computations of Deflated Vascular Geometries fo Fluid-Structure Interaction in Haemodynamics. PhD thesis, Università degli Studi di Napoli Federico II, July 2012.

[13] F. Nobile, M. Pozzoli, and C. Vergara. Inexact accurate partitioned algorithms for fluid-structure interaction problems with finite elasticity in haemodynamics. Journal of Computational Physics, 273:598-617, 2014.

[14] F. Nobile and C. Vergara. An effective fluid-structure interaction formulation for vascular dynamics by generalized Robin conditions. SIAM J Sc Comp, 30(2):731763, 2008.

[15] K. Perktold, E. Thurner, and T. Kenner. Flow and stress characteristics in rigid walled and compliant carotid artery bifurcation models. Medical and Biological Engineering and Computing, 32(1):19-26, 1994.

[16] P. Perona and J. Malik. Scale-space and edge detection using anisotropic diffusion. IEEE Transactions on Pattern Analysis and Machine Intelligence, 12(7):629639, 1990.

[17] A. Quarteroni, A. Manzoni, and C. Vergara. The cardiovascular system: Mathematical modelling, numerical algorithms and clinical applications. Acta Numerica, 26:365-590, 2017.

[18] S.G. Timsit, R.L. Sacco, J.P. Mohr, M.A. Foulkes, T.K. Tatemichi, P.A. Wolf, T.R. Price, and D.B. Hier. Early clinical differentiation of cerebral infarction from severe atherosclerotic stenosis and cardioembolism. Stroke, 23:486-491, 1992.

[19] M. Unser. Splines: a perfect fit for signal and image processing. IEEE Transactions on Signal Processing Magazine, 16(6):22-38, 1999.

[20] H.F. Younis, M.R. Kaazempur-Mofrad, R.C. Chan, A.G. Isasi, D.P. Hinton, A.H. Chau, L.A. Kim, and R.D. Kamm. Hemodynamics and wall mechanics in human carotid bifurcation and its consequences for atherogenesis: investigation of inter-individual variation. Biomechanics and Modeling in Mechanobiology, 3(1):17-32, 2004.

[21] Y. Yu, H. Baek, and G.E. Karniadakis. Generalized fictitious methods for fluidstructure interactions: Analysis and simulations. Journal of Computational Physics, 245:317-346, 2013.

[22] F. Zhu and J. Tian. Modified fast marching and level set method for medical image segmentation. J Xray Sci Technol, 11(4):193-204, 2003. 